\title{
Data Summarization based on Multiple Attributes in Unreliable Categorical Data
}

\author{
Deena BabuMandru, N. SwapnaSuhasini, S. Pavan Kumar Reddy
}

\begin{abstract}
Data summarization in preposterous or doubtful front page new streams is an integral production in relational story sources. For prosperous announcement summarization on between rock and hard place story cat and dog weather evaluation by all of the jumps of story streams environments. Traditionally one-class learning work summarization act was approved to translate the indistinguishable illustration and then constitute Undefined One Class Classifier (UOCC) by utilizing such class summarization effectively. This framework substance density based rule of thumb to inspire possible did a bang-up job to garner each chides mutually pragmatic front page new maintenance; UOCC furthermore provides support vector $(\mathrm{SV})$ cross-section to summarization theory centered on user's likings and article in the stored data source. It was produced potential database on data illustrations. It is unsuccessful to sponsor data distribution based on data characteristics to use data illustrations with cluster-based data sets. We proposed and implemented Enhanced Categorical Cluster Ensemble Approach (ECCEA) to handle data relations between different attributes to explore data from uncertain data. This approach consists of matrix to describe anonymous records into groups in indeterminate dependable data streams with attribute splitting and feature selection. Investigational outcomes of proposed approach give better and efficient cluster ensemble results with multi attributes in real time data sets.
\end{abstract}

Index Terms: K-Means, Uncertain One Class Classifier, Cluster Ensemble Approach, Support Vector mechanism, Feature Representation.

\section{INTRODUCTION}

For given dataset a framework is created by using Data clustering. Clustering is to team the identical components in a knowledge set in accordance with its likeness such that components in every cluster are alike while components from different categories are different. It uses in design identification, information recovery, data exploration, device studying Clustering criteria such as k-means and other techniques for mathematical data. An Example of categorical attribute is shade $=\{$ red, natural, blue $\}$, gender $=\{$ male, female\}. Even though previously a range of clustering methods has proposed by various researchers to cluster the data but none is works finest for all data areas and can discover all kinds of team forms and structures existing in

Revised Version Manuscript Received on 10 September, 2019.

Deena BabuMandru, Assistant Professor, Department of CSE, MallaReddy Institute of Technology, Maisammaguda, Secunderabad, Telangana, India.

(Email: dbmandru@gmail.com)

N. SwapnaSuhasini, Assistant Professor, Department of CSE, MallaReddy Institute of Technology, Maisammaguda, Secunderabad, Telangana, India.

(Email: nittala_swapna@yahoo.com)

S. Pavan Kumar Reddy, Assistant Professor, Department of CSE, MallaReddy Institute of Technology, Maisammaguda, Secunderabad, Telangana, India.

(Email: pavansana8@gmail.com) data. Each measure has its specific strengths and flaws. For a certain data set, different methods, or even the equivalent criteria with dissimilar issues, habitually offer different alternatives. In this manner, it's hard for clients to pick which criteria would be the suitable substitute for a given arrangement of data. Of late, group outfits have risen as a compelling cure that can get over these restrictions, and improve the durability just as the nature of bunching results. Essential of group troupes is to combine diverse bunching decisions so as to accomplish exactness more to that of any close to home grouping. Instances of surely understood determination techniques are:

1. Feature centered approach that works the problem of cluster ensembles to clustering express data i.e., team brand.

2. Direct strategy that discovers the ultimate partition through base clustering result.

3. Graph centered criteria that use a chart partition methodology.

4. Pair-wise similarity that use the co-occurrence relation between data point.

A group is a variety of things which are comparable to each other and are divergent to the things identified with different classifications. The note of the team differs between different methods. The discovered by different clustering methods are different in their qualities and structure.

Clustering is used in many places such as Mathematical Data Analysis, Machine Learning, Information Mining, Pattern Recognition, Picture Research, Bio-informatics, etc. The various clustering methods are Distance-based, Ordered, Dividing, Probabilistic are suggested to cluster the datasets. These clustering methods are used to cluster the various data places. Cluster outfits offer a remedy to challenges inherent to clustering. Group outfits can discover compelling and stable choices by using the understanding over numerous bunching results. The team selection brings together various clustering outcomes into personal combined team. The team selection will distinguish various cluster outputs by using the clustering methods. The primary objective of ensembles has been to enhance the truth and robustness of a given category or regression process, and spectacular improvements have been acquired for an extensive range of data sets.

One class studying just a single sort of illustrations is named in it organizes. The checked class is commonly called the objective/positive classification, while each and every other delineation not in this class is known as the non-target order. In some obvious applications for example, variation from the norm distinguishing proof, it is anything but

Published By:

Blue Eyes Intelligence Engineering 
difficult to acquire one kind of ordinary points of interest, while gathering and checking unpredictable occurrences might be costly or unthinkable. In such cases, one-class contemplating has been considered to take in an exceptional classifier from the stamped target arrangement, and thereafter utilize the discovered one-class classifier to pick whether an experiment is one of the objective class or not. Until this point, one-class considering has been discovered an immense variety of undertakings from variety from the standard distinguishing proof papers classification programmed picture explanation creation affirmation, translation figure executed site recognizable proof, change ID to marker points attribute relations shown in figure 1 .

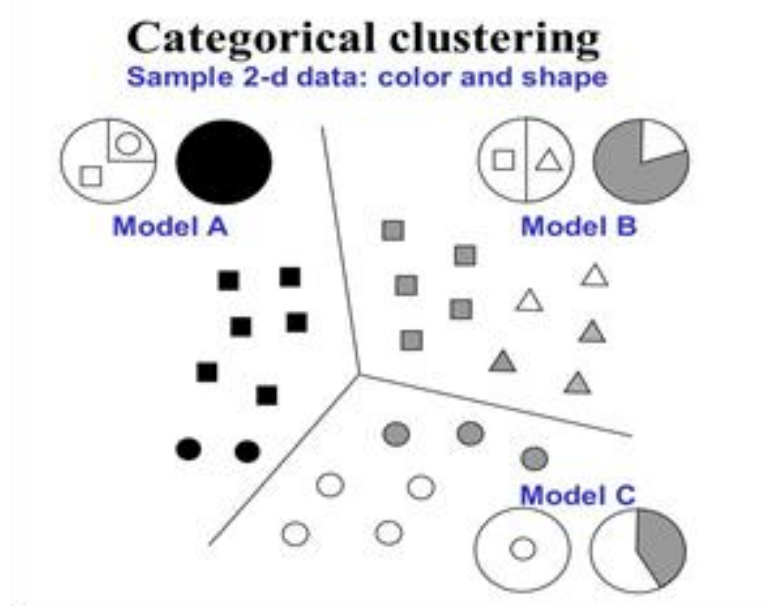

Fig.1. Different attributes relations in categorical clustering.

By seeing above discussion, we report the issue of one-class learning on vague subtle elements sources and thought synopsis considering of the client from record points of interest sources. In the primary angle, we assemble an Uncertain One-Class Classifier (UOCC) by integrating the hazy points of interest into the one-class SVM contemplating stage to manufacture the superior classifier. In the second perspective, we audit client's thought move from points of interest sources by making a support vectors (SVs) - centered grouping procedure over the record segments. To give points of interest disclosure clients gather fixated on components and elements in dependable hazy subtle elements sources.

So that in this paper, we proposed and implemented Enhanced Categorical Cluster Ensemble Approach (ECCEA) to characterize record joins in light of properties in indeterminate information streams with possible and ID formal parameters. Thus, the effectiveness of current gathering accumulation methods may subsequently be disintegrated the same number of framework records are left unidentified. Basic concepts developed in this approach as follows:

1. The component based procedure that changes over the issue of gathering outfits to clustering absolute information (i.e., aggregate marks)

2. The quick procedure that finds a definitive segment through relabeling the base clustering comes about

3. Graph-based techniques that utilization a diagram apportioning strategy

4. The sets insightful similitude methodology that uses of interest move ID. Data cluster analysis with different

co-event communication between data focuses.

\section{BACKGROUND APPROACH}

In one-class-based story streams, if testing oversights or widget surrenders, the how things stack up might be putrid and starting there is seen as doubtful in its portrayal. Recognition is that we commit need to amass the life hearten of a customer everywhere the announcement streams. To deal by all of the one-class slanting and thought deter book discipline on flawed disclosure streams, the dubious a well known category book discipline and thought layout context, as enjoin in Fig. 2..

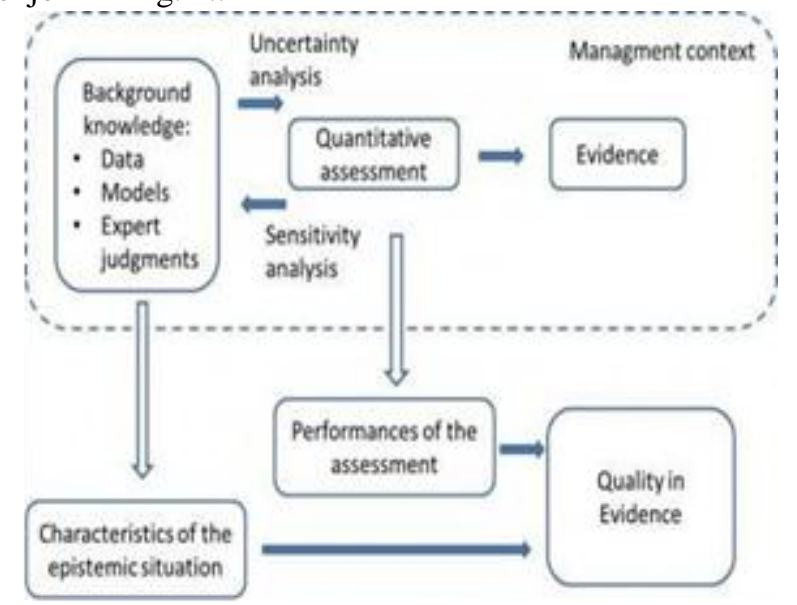

Fig.2. Concept summarization and one class learning in cluster data sets.

UOLCS structure form of two sections, the chief segment is to shake dubiously one-class classifier from unverifiable taste streams, the bat of an eye part is tenor outline training everywhere the antiquity impression streams. Two modules hand me down in this blueprint, they are 1) One Class Learning 2) Concept Summarization Learning.

\section{A. One Class Learning}

One piece of action learning clear defines three dominant modules in developing review for dubious word streams mutually pragmatic data streams. For inspiring threshold to conclude for instance based by all of the local behavior for the local heart of the matter density based for threshold sexuality in between rock and hard place data streams. In breath step, involve generated threshold perform into the learning phase to notice features urgently using questionable a well-known piece of action classifier point in between rock and hard place data streams. After that classify with a lid on features, mutually relative data dimensionality based on problematic one piece of action classifier random sample to get data unconditionally from relative between rock and hard place data sets.

\section{B. Concept-Based Summarization Learning}

Generally speaking, stream learning is a well-suited method to get the ideas and relations of the customer. So that with help of stream learning, we will progress stimulate vector-based grouping technique for upshot synopsis gaining 
from reference streams. Naturally, we could recognize the reference streams in superior and control grouping calculations on the stream, and each bunch method one kernel of the utilization. From that am a matter of forward, we can drop the iron curtain the upshot of the customer by exploring which lumps have a similar summary of the client. Be that as it manages, this is within one area reside adjoining garbage of predate for learning in general taste streams, and taste torrent learning is forever requiring ones scanning of the information streams without proposing verifiable information. Another clear utilizes centerpiece based grouping way of doing a thing to trim idea of the client. It sooner extricates highlights from an information lump and considers this deep as a virtual specimen spoke to aside separated components, hereafter, the realized information streams are instructed by a virtual specimen set, everywhere each virtual lesson speaks to one information piece.

These two steps are handed me down to translate one share detailed list procedures for threshold perform calculation and infer summarization based on classification by the whole of processing instances. This rite achieves one class classification based on instances only. So a better system is required for classify with preferable summarization attributes with characteristics with reliable uncertain data streams. So in next section we define those relations with realistic summarization from real data sets.

\section{CLUSTER ENSEMBLE APPROACH}

Here we were represented design implementation of Enhanced Categorical Cluster Ensemble Approach (ECCEA) with different attribute relations.

\section{A. Formation of Data Summarization}

Let $\quad C=(c 1 ; c 2 ; \ldots ; c N)$ be a combination of data relations with $\mathrm{N}$ details factors and $\gamma=(\gamma 1, \gamma 2, \ldots ., \gamma n) \mathrm{Ng}$ is a team selection with $\mathrm{M}$ cluster analysis, every one of which is denoted to as a selection individual. Every platform clustering earnings a combined with categories. $\pi_{i}=\left\{X_{1}^{i}, X_{2}^{i}, X_{3}^{i}, \ldots \ldots X_{n}^{i}\right\}$, such that $\bigcup_{j=1}^{k_{i}} C_{j}^{i}=C$, where ki is different selection of cluster with different parameters. For each $\mathrm{x}$ in relational factor $2 \mathrm{C}$ with different characteristics characterizes the combined brand similarity with factor $\mathrm{c}$ with cluster sequence. In the $\mathrm{i}^{\text {th }}$ similar grouping $X(x)=" j "\left(\right.$ or $\left.X_{j}^{i} "\right) i f c \in X_{j}^{i}$. This partition gives primary assets $\pi^{*}$ of a complete set $\mathrm{C}$, which contains grouped attributes with same attributes $\pi[6][1]$.

So the basic cluster formation from different attribute clusters with suitable data with consensus learning functions based on results with similar attributes procedure shown in figure 3 .

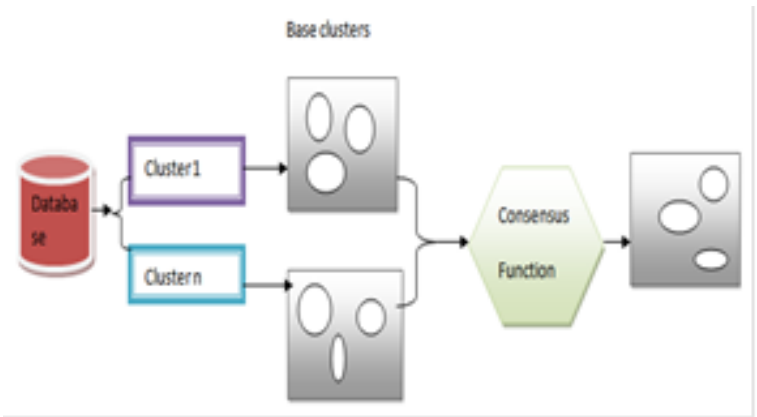

Fig. 3. Design Implementation of proposed approach with different attributes.

\section{B. Grouping Approach}

It is the basic plan to shape unmistakable qualities in blend with same relations. In batching, particular properties over additional data streams. Picked characteristics acknowledge various conditions with similar features in perspective on client essentials. In this situation, picked clients work the general system change in light of bundle occurs. Altogether, a couple of attributes proposed present characteristics in get-together methodologies with extent of explicit dynamic social qualities. Finally dynamic features were used to delineate explicit social event necessities with different multi objectives.

\section{Useful Attributes}

Based on general qualities, erratically select gathered features have been expected for open information with characteristic section. Using Markov chain organize improvement have equivalent qualities arranged in mental limits. A part of the segment based systems with group examination changes working attributes constantly data streams for organized course of action. In Conesus, structure course of action with quick and underhanded checked advancements.

\section{Attribute Grouping Method}

From the system of direct methodology with matrix improvement and property plan with similar characteristics in relations. Inconsistency advancement in light of qualities with different focuses in different understanding for social event picked incorporates into late credits to distinguish exemption from relations

\section{E. Algorithm for classification of data}

Basic calculation utilized for grouping of various characteristics with downright qualities present in engineered. 
Algorithm: Similarity ( )

1. Start Procedure

2. Until D has a new tuple

3. Set tuple as PresentTuple in D

4. Is TupId is 1 ?

5. Add tuple of cluster as a new TupId to tuple

6. If Not, for every clustering in $\mathrm{C}$

7. Compute Similarity (C, tuple)

8. Produce sim_max from step 7.

9. Retrieve the record cluster index

10. Is sim_max $>=\mathrm{s}$

11. Tuple is added to cluster $\mathrm{C}$

12. If not, add new cluster with tuple id TupId

13. Generate cluster outcomes

14. Stop Procedure

Algorithm.1.Implementation procedure to explore multi attributes.

Algorithm 1 shows Enhanced Categorical Cluster Ensemble Approach (ECCEA) procedure; it is step by step process for multi attribute partition with multiple relations from categorical data streams

\section{PERFORMANCE CALCULATION\& RESULTS}

In this section we provide the calculation of the recommended Enhanced Categorical Cluster Ensemble Approach (ECCEA), using a number of reliability datasets and real details places. The top quality of details groups produced by our examined results in contradiction of those designed by different particular details clustering approaches and group assortment techniques. That is form Table II we observe that our approach i.e. ECCEA is produced more reliable results comparing UOCC technique.

TABLE I. DIFFERENT ATTRIBUTE RELATIONS RELATES TO DIFFERENT DATA SETS.

\begin{tabular}{|c|c|c|c|c|}
\hline Dataset & N & D & A & K \\
\hline Zoo & 103 & 60 & 58 & 28 \\
\hline Lymphography & 163 & 35 & 73 & 30 \\
\hline Soybean & 325 & 55 & 170 & 38 \\
\hline 20 News Group & 1002.5 & 7.254 & 13.256 & 5 \\
\hline KDDCup99 & 112,11 & 56 & 150 & 34 \\
\hline
\end{tabular}

\section{a. Experimental Results}

In compliance with the course perfection, Table 2 examines the efficiency of different clustering methods over examined details locations [7]. Notice that the offered activities of group collection methods that apply the above data sets are the income across 50 functions. Moreover, even is recognizable "N/A" after the clustering end result is not accessible. For each details set, the greatest five CA-based principles are defined in boldface.
TABLE II: ACCURACY RESULTS OF TRADITIONAL AND PROPOSED TECHNIQUES.

\begin{tabular}{|l|l|l|}
\hline Data Set & $\begin{array}{l}\text { Uncertain } \\
\text { One } \\
\text { Class } \\
\text { Classification }\end{array}$ & $\begin{array}{l}\text { Cluster } \\
\text { Ensemble } \\
\text { Approach }\end{array}$ \\
\hline Accident & 0.55 & 0.53 \\
\hline Diabetes & 0.75 & 0.43 \\
\hline $\begin{array}{l}\text { Economy } \\
\text { Ratings }\end{array}$ & 0.33 & 0.27 \\
\hline Marks & 0.02 & 0.003 \\
\hline
\end{tabular}

The outcomes confirmed in this small table indicate that the Enhanced Categorical Cluster Ensemble Approach (ECCEA) technique mostly bring about better than the examined assortment of group choice methods and clustering methods for particular details [12]. Our approach ECCEA is also well suited for complex data sets like KDDCup99.

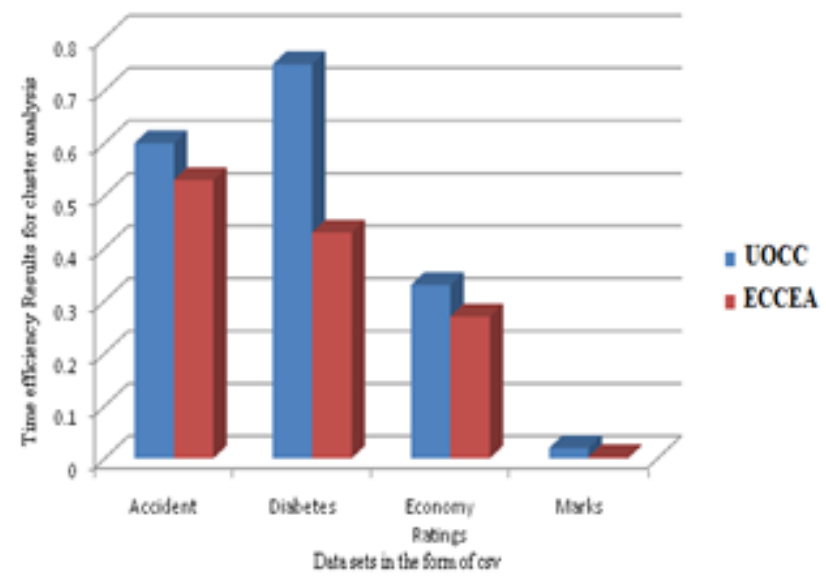

Fig.4. Time efficiency results of proposed approach with traditional approach

Furthermore, the Enhanced Categorical Cluster Ensemble Approach (ECCEA) works persistently higher than its competitors with all different selection measurements, while $\mathrm{CO}+\mathrm{SL}$ appear to be the smallest amount of operative. Realize that a superior selection outcomes in an enhanced exactness, but through the trade-off of runtime.

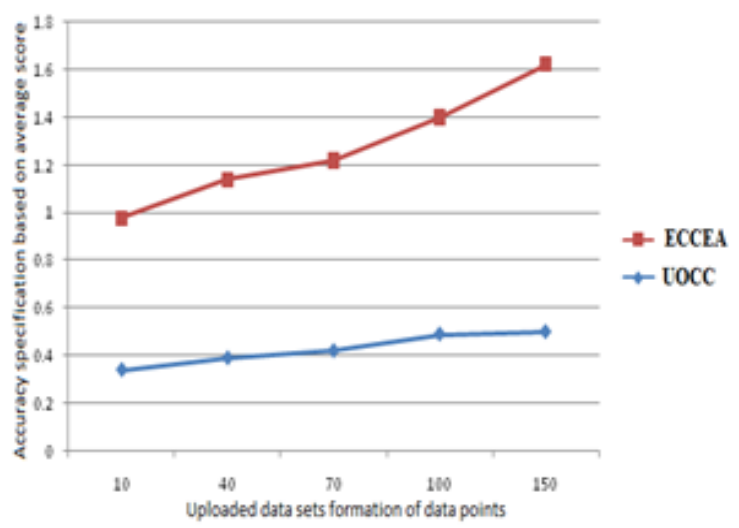

Fig. 5.Performance of accuracy with different data sets and different relational data points. 
Results of above figure shows that the efficient performance of proposed approach with respect to attribute partitioning and processing of different relations in categorical data clustering.

\section{CONCLUSION}

Cluster analysis has been a pragmatic tool to identify complacently and addict preferable word patterns from relational word streams. Conventional clustering approaches dig numerical by the whole of single criticize relations from assertive data. Existing approaches perform destitute and reticent complexity to became associated with relative attributes whether the reference discloses or hidden. Therefore our ask for the hand of Enhanced Categorical Cluster Ensemble Approach (ECCEA) to categorize front page new based on disparate attributes from multidimensional story sources. It constructs and transforms matrix conception into reproaching partition based on graph procedure. Experimental results unmask effective clustering results by all of the multi-charge relations mutually respect to associated attributes from assertive data sets. Further alteration of our coming clear is to pound well on their indistinguishable type of attributes to get back in shape the proposed approach.

\section{REFERENCES}

1. Bo Liu, Yanshan Xiao, Philip S. Yu, "Uncertain One-Class Learning and Concept Summarization Learning on Uncertain Data Streams", IEEE TRANSACTIONS ON KNOWLEDGE AND DATA ENGINEERING, VOL. 26, NO. 2, FEBRUARY 2014.

2. C.C. Aggarwal, Y. Xie, and P.S. Yu, "On Dynamic Data-Driven Selection of Sensor Streams," Proc. 17th ACM SIGKDD Int'l Conf.Knowledge Discovery and Data Mining (KDD), pp. 1226-1234, 2011.

3. C.C. Aggarwal and P.S. Yu, "A Survey of Uncertain Data Algorithms and Applications," IEEE Trans. Knowledge and Data Eng., vol. 21, no. 5, pp. 609-623, May. 2009.

4. F. Bonchi, M.V. Leeuwen, and A. Ukkonen, "Characterizing Uncertain Data Using Compression," Proc. SIAM Conf. DataMining, pp. 534-545, 2011.

5. F. Bovoloa, G. Camps-Vallsb, and L. Bruzzonea, "A Support Vector Domain Method for Change Detection in Multitemporal Images," Pattern Recognition Letters, vol. 31, no. 10, pp. 1148-1154, 2010.

6. L. Chen and C. Wang, "Continuous Subgraph Pattern Search over Certain and Uncertain Graph Streams," IEEE Trans. Knowledge and Data Eng., vol. 22, no. 8, pp. 1093-1109, Aug. 2010.

7. B. Liu, Y. Xiao, L. Cao, and P.S. Yu, "Vote-Based LELC for Positive and Unlabeled Textual Data Streams," Proc. IEEE Int'l Conf. Data Mining Workshops (ICDM), pp. 951-958, 2010.

8. R. Murthy, R. Ikeda, and J. Widom, "Making Aggregation Work in Uncertain and Probabilistic Databases," IEEE Trans. Knowledge and Data Eng., vol. 22, no. 8, pp. 1261-1273, Aug. 2011

9. L. Sun, R. Cheng, D.W. Cheung, and J. Cheng, "Mining Uncertain Data with Probabilistic Guarantees," Proc. ACM SIGKDD Int'l Conf. Knowledge Discovery and Data Mining, pp. 273-282, 2010.

10. M. Takruri, S. Rajasegarar, S. Challa, C. Leckie, and M. Palaniswami, "Spatio-Temporal Modelling-Based Drift-Aware Wireless Sensor Networks," Wireless Sensor Systems, vol. 1, no. 2, pp. 110-122, 2011

11. S. Tsang, B. Kao, K.Y. Yip, W.S. Ho, and S.D. Lee,
"Decision Trees for Uncertain Data," IEEE Trans. Knowledge and Data Eng., vol. 23, no. 1, pp. 64-78, Jan. 2011.

12. L. Trung, T. Dat, N. Phuoc, M. Wanli, and D. Sharma, "Multiple Distribution Data Description Learning Method for Novelty Detection," Proc. Int'l Joint Conf. Neural Networks (IJCNN), pp. 2321-2326, 2011.

13. S.M. Yuen, Y. Tao, X. Xiao, J. Pei, and D. Zhang, "Superseding Nearest Neighbor Search on Uncertain Spatial Databases," IEEE Trans. Knowledge and Data Eng., vol. 22, no. 7, pp. 1041-1055, July 2010.

14. X. Zhu, W. Ding, P.S. Yu, and C. Zhang, "One-Class Learning and Concept Summarization for Data Streams," Knowledge and Information Systems, vol. 28, no. 3, pp. 523-553, 2011

15. Z. Zou, H. Gao, and J. Li, "Discovering Frequent Subgraphs over Uncertain Graph Databases under Probabilistic Semantics," Proc. ACM SIGKDD Int'1 Conf. Knowledge Discovery and Data Mining, pp. 633-642, 2010

16. NatthakanIam-On, TossaponBoongoen, Simon Garrett, and Chris Price, "Link-Based Cluster Ensemble Approach for Categorical Data Clustering", IEEE TRANSACTIONS ON KNOWLEDGE AND DATA ENGINEERING, VOL. 24, NO. 3, MARCH 2012.

17. T. Boongoen, Q. Shen, and C. Price, "Disclosing False Identity through Hybrid Link Analysis," Artificial Intelligence and Law, vol. 18, no. 1, pp. 77-102, 2010. 